\title{
A case of positive HIV serology revealed by a demodicosis
}

\section{Ibtissam Assenhaji, Mounia Bennani, Sara Elloudi, Hanane Baybay, Fatima Zahra Mernissi}

\author{
Dermatology Department, Fes Hospital Center, Fes, Morocco \\ Corresponding author: Dr. Ibtissam Assenhaji, E-mail: assenhaji.louizi@outlook.fr
}

\begin{abstract}
Demodicosis is a cutaneous opportunistic ectoparasitic infection, caused by the proliferation of Demodex in the pilosebaceous apparatus mainly on the face, near the nose, the commissures of the lips, eyelashes and eyebrows.

The Demodicosis is in association with acquired deficiency syndrome (AIDS), diabetes and haematological malignancies. The clinical expression of the Demodicosis is polymorphic and sometimes misleading, diagnosis is made by scraping the scales with a curette, or a biopsy. The treatment consists of topical or general metronidazole. We report the case of Demodicosis revealing positive HIV serology on a pregnant woman.
\end{abstract}

Key words: Demodicosis; Positive HIV serology; Demodex

\section{INTRODUCTION}

Demodicosis is a cutaneous opportunistic ectoparasitic infection, common to humans and many mammals, caused by the proliferation of Demodex in the pilosebaceous apparatus [1]. The parasite is very common in the face, but it can be found in other seborrheic areas of the body.

We report the case of Demodicosis revealing positive HIV serology on a pregnant woman.

\section{CASE REPORT}

This is a 28-year-old patient, pregnant at 16 week, having a history of recurrent herpes labialis, genital warts. Who presents since 3 months an erythematous pruriginous lesions in the face, the upper back, and trunk. Without risky sexual behavior, transfusion, or drug addiction.

The dermatological examination found erythematous papules in the cheeks, the forehead, the upper part of the thorax, scattered with pustules (Figs 1 and 2), the dermoscopy showed erythema strewn with telangiectasia, a demodex aspect of the follicular ostium (Figs. 3 and 4). The rest of the somatic examination was normal.

The patient received a sample of scales showing the presence of demodex, with positive HIV serology.

The patient was treated with erythromycin at a dose of $500 \mathrm{mg} /$ day, and a copper zinc cream, an antiviral treatment, with a good improvement.

\section{DISCUSSION}

Demodex are common and permanent ectoparasitic mites in humans. They are saprophytes of the pilosebaceous follicle and are part of the microbiota of the skin. They are naturally present on or in the skin of human beings [2], mainly on the face, near the nose, the commissures of the lips, eyelashes and eyebrows, in the sebaceous glands of the face [1].

The Demodicosis is described above in association with acquired deficiency syndrome (AIDS), diabetes and haematological malignancies [1], Nevertheless, under

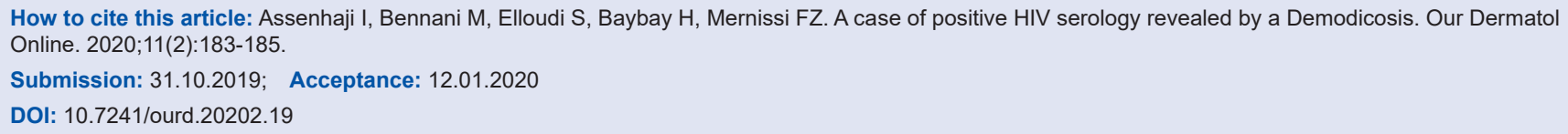




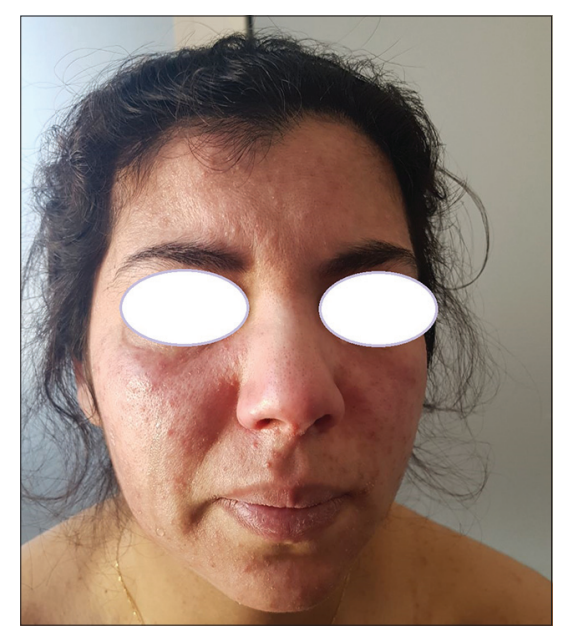

Figure 1: Erythematous papules in the cheeks, forehead, upper chest.

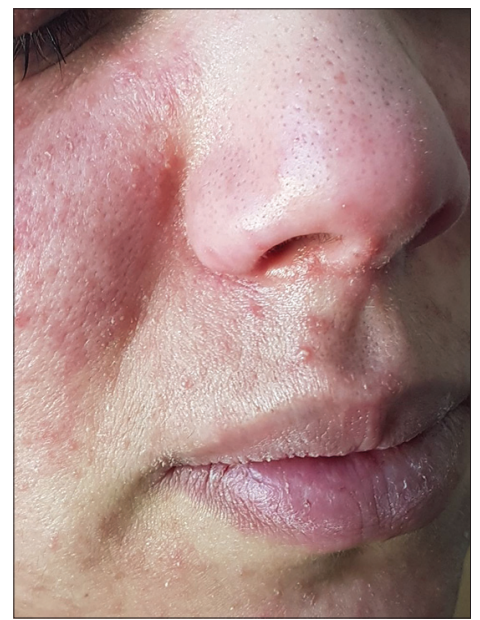

Figure 2: Presence of pustules in the cheeks of the upper lip and chin.

certain conditions, these might sometimes play a role in certain dermatological pathologies (rosacea, pustular folliculitis, blepharitis) [2].

The clinical expression of the Demodicosis is polymorphic and sometimes misleading, made of erythematous and papulopustular lesions pruriginous of chronic and recurrent evolution [3].

The paraclinical diagnosis is made by scraping the scales with a curette, or a biopsy to visualize the inflammatory reaction and to detect the parasite.

The treatment consists of daily washing, associated with topical metronidazole for several weeks, the eviction of fat creams and local corticosteroids. General treatments (metronidazole, ivermectin, cyclins) [4,5], can be combined with local treatments, especially in immunodepressed patients.

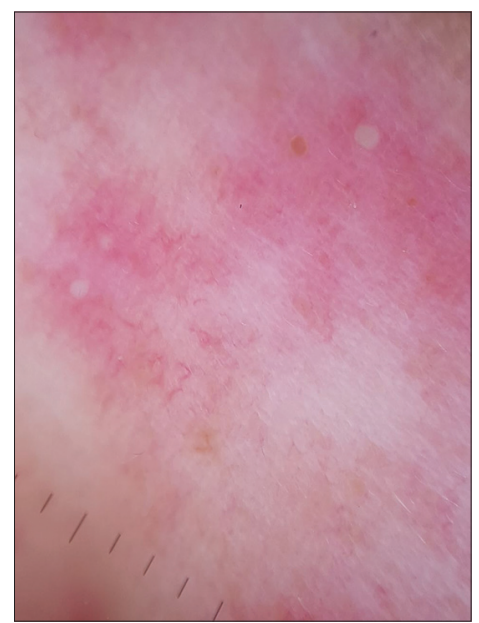

Figure 3: Dermoscopy presence of erythema strewn with telangiectasia, a pustules.

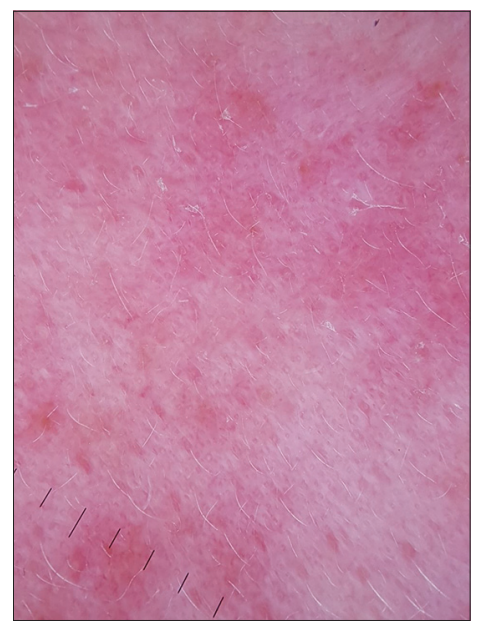

Figure 4: Dermoscopy presence of, a demodex aspect of the follicular ostium.

\section{CONCLUSION}

The clinical expression of Demodicosis is polymorphous and sometimes misleading, may reveal an HIV infection. Parasitological confirmation is essential. Treatment with metronidazole seems to be effective.

\section{Consent}

The examination of the patient was conducted according to the Declaration of Helsinki principles.

The authors certify that they have obtained all appropriate patient consent forms. In the form the patient(s) has/have given his/her/their consent for his/her/their images and other clinical information to be reported in the journal. The patients understand that their names and initials will not be published and 
due efforts will be made to conceal their identity, but anonymity cannot be guaranteed.

\section{REFERENCES}

1. Bouattour Y, Rekik F, Jallouli M, Garbaa S, Turki C, Frikha F, et al. Démodécidose faciale mimant une poussée d'un lupus érythémateux systémique. J. Rev Med. 2016;10:334.

2: Ozdemir MH, Aksoy U, Sonmez E, Akisu C, Yorulmaz C, Hilal A. Prevalence of Demodex in health personnel working in the autopsy room. Am J Forensic Med Pathol. 2005;26:18-23.

3. Neji S, Cheikhrouhou F, Trabelsi H, Sellami H, Boudaya S,
Sellami $\mathrm{D}$, et al. Les démodécidoses: à propos de 719 cas. J Ann Dermatol. 2012;10:417.

4. Dirk M, Elsion MD. Demodex mites; Facts and contorvesies. Clin Dermat. 2010:28:502-4.

5. El-Shazly AM, Hassan AA, Soliman M, Morsy GH, Morsy TA. Treatment of human Demodex folliculorum by camphor oil and metronidazole. J Egypt Soc Parasitol. 2004;34:107-16.

Copyright by Ibtissam Assenhaji, et al. This is an open-access article distributed under the terms of the Creative Commons Attribution License, which permits unrestricted use, distribution, and reproduction in any medium, provided the original author and source are credited. Source of Support: Nil, Conflict of Interest: None declared. 